archives

of thermodynamics

Vol. 31(2010), No. 3, 19-36

DOI: $10.2478 / \mathrm{v} 10173-010-0012-\mathrm{y}$

\title{
Modelling of heat and flow phenomena occuring in waterwall tubes of boilers for supercritical steam parameters
}

\author{
WIESŁAW ZIMA* \\ SŁAWOMIR GRĄDZIEL \\ ARTUR CEBULA
}

Cracow University of Technology, Department of Thermal Power Engineering, al. Jana Pawła II 37, 31-864 Kraków, Poland

\begin{abstract}
In this paper a mathematical model enabling the analysis of the heat-flow phenomena occurring in the waterwalls of the combustion chambers of the boilers for supercritical parameters is proposed. It is a onedimensional model with distributed parameters based on the solution of equations describing the conservation laws of mass, momentum, and energy. The purpose of the numerical calculations is to determine the distributions of the fluid enthalpy and the temperature of the waterwall pipes. This temperature should not exceed the calculation temperature for particular category of steel. The derived differential equations are solved using two methods: with the use of the implicit difference scheme, in which the mesh with regular nodes was applied, and using the Runge-Kutta method. The temperature distribution of the waterwall pipes is determined using the CFD. All thermophysical properties of the fluid and waterwall pipes are computed in real-time. The time-spatial heat transfer coefficient distribution is also computed in the on-line mode. The heat calculations for the combustion chamber are carried out with the use of the zone method, thus the thermal load distribution of the waterwalls is known. The time needed for the computations is of great importance when taking into consideration calculations carried out in the on-line mode. A correctly solved one-dimensional model ensures the appropriately short computational time.
\end{abstract}

Keywords: Waterwalls; Supercritical parameters; Mathematical model; Distributed parameters; Implicit difference scheme

\footnotetext{
*Corresponding author. E-mail address: zima@mech.pk.edu.pl
} 


\section{Nomenclature}

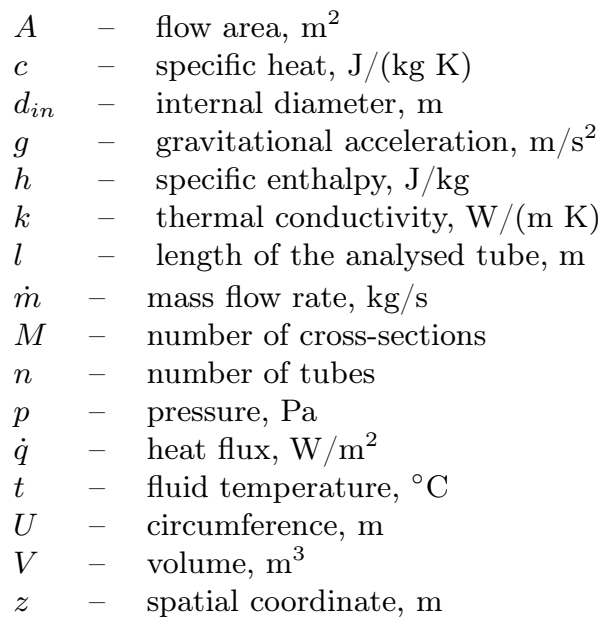

\section{Greek symbols}

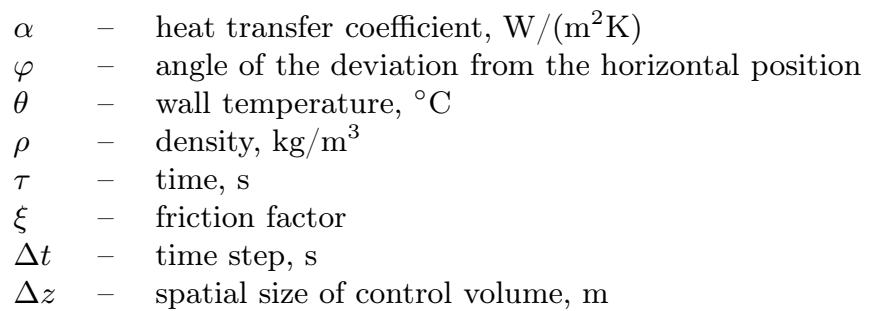

\section{Introduction}

Problems concerning modelling of the transient heat-flow processes occurring in the heated surfaces of power boilers raised and described in both domestic and foreign literature indicate their complexity and strong nonlinearity [1-8]. The complexity of the analysis of these processes is caused by high temperature and pressure values as well as large heat-exchange surfaces and their fouling. The nonlinearity results mainly from the dependency of thermophysical properties of the fluid on the temperature and pressure. These properties vary significantly especially near the critical point. The diagnosis of heat-flow processes in the power engineering industry is based on thermally steady processes. This comes from the lack of mathematical models of large thermally unsteady energy objects [9]. 
In this paper, a mathematical model enabling the analysis of the heatflow phenomena occurring in boiler combustion chamber waterwalls for supercritical parameters is proposed. This is a one-dimensional model with distributed parameters, based on the solution of equations describing the conservation laws of mass, momentum, and energy.

On the basis of the above mentioned equations the distribution of the fluid enthalpy in the combustion chamber pipe system is determined. Knowing the fluid temperature the waterwall pipes temperature can be determined. This is of essential importance to avoid the overheating of the pipe system, especially in the upper sections of the waterwalls. The temperature distribution of the waterwall pipe, together with the fin, is determined with the use of the computational fluid dynamics (CFD) software [10].

Two methods of solving the equations describing the laws of mass, momentum, and energy conservation are proposed. In the first one the implicit finite difference scheme is applied. The second method for solving the balance equations is the Runge-Kutta method. The accuracy and stability of the numerical calculations, carried out with the use of difference schemes, depend on the assumed for this calculations time-spatial steps. These steps are evaluated based on a comparison of the results obtained using the proposed method with the results of analytical solutions for transient states.

\section{Description of the mathematical model}

A mathematical model for simulating the heat-flow phenomena occurring in the waterwalls of combustion chambers in boilers operating with supercritical parameters is proposed. It is a one-dimensional model with distributed parameters based on the solution of equations describing the conservation laws of mass, momentum, and energy. The purpose of this calculation is determination of the enthalpy, mass flow rate, and the pressure distributions of the fluid in the boiler combustion chamber pipes.

For the modelling of the heat-flow processes in continuous media, the conservation equations of mass, momentum, and energy in differential form are used [11]. The control volume (CV) of the waterwall pipe, shown in Fig. 1, is analysed. The equations are for the single-phase flow along the $z$ axis.

The principle of conservation of any arbitrary $Q$ value for a given control volume is taken into consideration: 


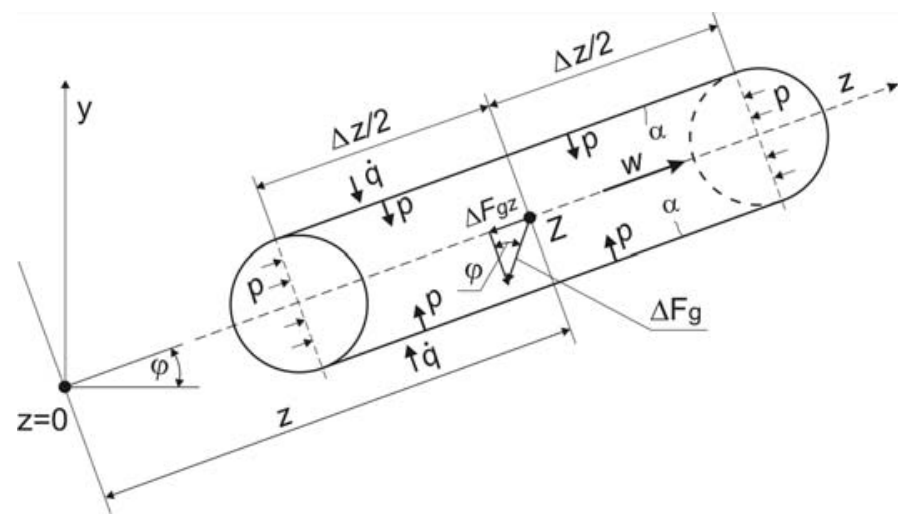

Figure 1. Analysed control volume of length $\Delta z$.

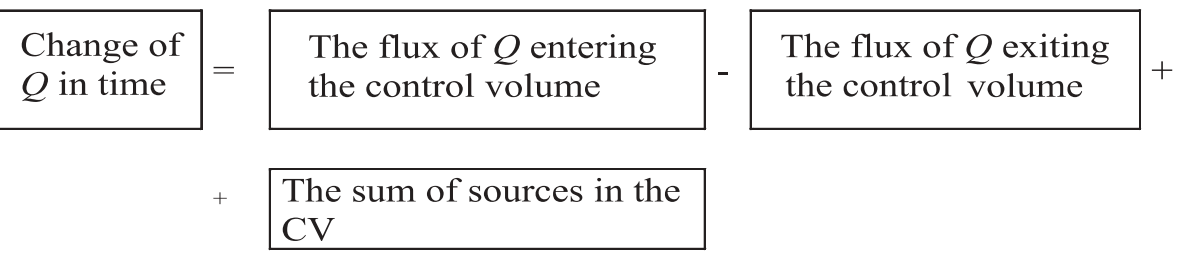

\subsection{The mass conservation equation}

The equation representing the mass balance takes the following form (Fig. 1):

$$
\frac{\partial(\Delta V \rho)}{\partial \tau}=\left.(A \rho w)\right|_{z-\Delta z / 2}-\left.(A \rho w)\right|_{z+\Delta z / 2}
$$

After some transformation:

$$
\frac{\partial \rho}{\partial \tau}=-\frac{1}{A} \frac{\partial \dot{m}}{\partial z}
$$

\subsection{The momentum conservation equation}

In the momentum balance equation for the control volume, apart from the momentum of both inflow and outflow flux, as well as the rate of changes in the momentum accumulated in the control volume, all forces affecting the control volume are taken into consideration. These forces can be divided into surface and mass forces. Thus the principle of momentum conservation 
can be expressed as:

$$
\begin{gathered}
\frac{\partial}{\partial \tau}(A \Delta z \rho w)=\left.(\dot{m} w)\right|_{z-\Delta z / 2}-\left.(\dot{m} w)\right|_{z+\Delta z / 2}+ \\
+A\left(\left.p\right|_{z-\Delta z / 2}-\left.p\right|_{z+\Delta z / 2}\right)-\sigma_{\tau} U \Delta z-\Delta V \rho g \sin \varphi
\end{gathered}
$$

After rearranging, equation (3) takes the following form:

$$
\frac{\partial \dot{m}}{\partial \tau}=-\frac{1}{A} \frac{\partial}{\partial z}\left(\frac{\dot{m}^{2}}{\rho}\right)-A\left(\frac{\partial p}{\partial z}+\frac{\partial p_{\tau}}{\partial z}+\rho g \sin \varphi\right) .
$$

\subsection{The energy conservation equation}

The energy balance for the control volume is as follows:

$$
\begin{array}{r}
\frac{\partial}{\partial \tau}(\Delta V \rho e)=A\left(\left.\rho w e\right|_{z-\Delta z / 2}-\left.\rho w e\right|_{z+\Delta z / 2}\right)+\dot{q} U \Delta z+ \\
+A\left[-\left.k \frac{\partial t}{\partial z}\right|_{z-\Delta z / 2}-\left.\left(-k \frac{\partial t}{\partial z}\right)\right|_{z+\Delta z / 2}\right]+A\left(\left.p w\right|_{z-\Delta z / 2}-\left.p w\right|_{z+\Delta z / 2}\right) .
\end{array}
$$

The first term (first bracket) on the right hand side of the Eq. (5) stands for the difference of the total convective energy flux flowing in and out from the control volume. The second term represents the heat flow rate into the CV through its surface $U \Delta z$. The third term of the right hand side of the Eq. (5) (second bracket) stands for the difference of the heat flux transferred and carried away from the control area by conduction. The last bracket represents the change of the pressure force during operation (the difference of the compression power of the fluid supplied and carried away from the $\mathrm{CV}$ ).

In Eq. (5) the work (energy) carried out by the volume $\dot{q}_{v} \Delta V$ and surface forces are omitted. After some transformation the following equation is obtained:

$$
\frac{\partial h}{\partial \tau}=\frac{\dot{m}}{A \rho}\left(\frac{1}{\rho} \frac{\partial p}{\partial z}-\frac{\partial h}{\partial z}+\frac{1}{\rho} \frac{\partial p_{\tau}}{\partial z}\right)+\frac{4 \alpha(\theta-t)}{d_{i n} \rho}+\frac{1}{\rho} \frac{\partial}{\partial z}\left(k \frac{\partial t}{\partial z}\right)+\frac{1}{\rho} \frac{\partial p}{\partial \tau} .
$$

Substituting the pressure derivative with respect to time:

$$
\frac{\partial p}{\partial \tau}=\frac{\partial p}{\partial \rho} \frac{\partial \rho}{\partial \tau}+\frac{\partial p}{\partial h} \frac{\partial h}{\partial \tau}=-\frac{1}{A} \frac{\partial p}{\partial \rho} \frac{\partial \dot{m}}{\partial z}+\frac{\partial p}{\partial h} \frac{\partial h}{\partial \tau}
$$


and also omitting the heat conduction in the fluid, the following final form of the energy balance equation is obtained:

$$
\frac{\partial h}{\partial \tau}=\left(1-\frac{1}{\rho} \frac{\partial p}{\partial h}\right)^{-1}\left[\frac{\dot{m}}{A \rho}\left(\frac{1}{\rho} \frac{\partial p}{\partial z}-\frac{\partial h}{\partial z}+\frac{1}{\rho} \frac{\partial p_{\tau}}{\partial z}\right)+\frac{4 \alpha(\theta-t)}{d_{i n} \rho}-\frac{1}{A \rho} \frac{\partial p}{\partial \rho} \frac{\partial \dot{m}}{\partial z}\right] .
$$

The pipe wall temperature $\theta$ in the Eq. (8) is determined from the equation of transient heat conduction [2].

\section{Solutions of the balance equations}

Presented below are two methods of solving the balance equations (2), (4), and (8).

\subsection{Method 1}

In this section, the expressions are derived for determining the time-spatial distributions of the mass flow rate, pressure and enthalpy of the fluid. On the basis of determined pressure and enthalpy distributions, the fluid density and temperature are evaluated. For the purpose of solving Eqs. (2), (4) and (8), the implicit difference method is used. The spatial mesh with regular nodes is applied.

Once the time derivatives are replaced by forward difference scheme and the spatial derivatives by backward difference scheme, the formulae (2), (4) and (8) take the form of Eqs. (9), (10) and (12). They allow determination of the time-spatial distributions of the mass flow rate, pressure and enthalpy of the fluid, respectively ( $M$ denotes the number of analysed cross-sections):

$$
\begin{gathered}
\dot{m}_{j}^{\tau+\Delta \tau}=\dot{m}_{j-1}^{\tau+\Delta \tau}+A \Delta z \frac{\rho_{j}^{\tau}-\rho_{j}^{\tau+\Delta \tau}}{\Delta \tau}, j=2, \ldots, M \\
p_{j}^{\tau+\Delta \tau}=p_{j-1}^{\tau+\Delta \tau}+\frac{\Delta z}{A \Delta \tau}\left(\dot{m}_{j}^{\tau}-\dot{m}_{j}^{\tau+\Delta \tau}\right)-\frac{1}{A^{2}}\left[\frac{\left(\dot{m}_{j}^{\tau+\Delta \tau}\right)^{2}}{\rho_{j}^{\tau+\Delta \tau}}-\frac{\left(\dot{m}_{j-1}^{\tau+\Delta \tau}\right)^{2}}{\rho_{j-1}^{\tau+\Delta \tau}}\right]- \\
-\left.\Delta z \frac{\partial p_{\tau}}{\partial z}\right|_{j} ^{\tau+\Delta \tau}-\Delta z \rho_{j}^{\tau+\Delta \tau} g \sin \varphi, \quad j=2, \ldots, M
\end{gathered}
$$

where:

$$
\left.\frac{\partial p_{\tau}}{\partial z}\right|_{j} ^{\tau+\Delta \tau}=\frac{\xi_{j}^{\tau+\Delta \tau}\left|\dot{m}_{j}^{\tau+\Delta \tau}\right| \dot{m}_{j}^{\tau+\Delta \tau}}{2 d_{i n} A^{2} \rho_{j}^{\tau+\Delta \tau}}
$$




$$
\begin{aligned}
h_{j}^{\tau+\Delta \tau}=h_{j}^{\tau}+ & \Delta \tau\left(1-\left.\frac{1}{\rho_{j}^{\tau}} \frac{\partial p}{\partial h}\right|_{j} ^{\tau}\right)^{-1}\left[\frac { \dot { m } _ { j } ^ { \tau } } { A \rho _ { j } ^ { \tau } } \left(\frac{1}{\rho_{j}^{\tau}} \frac{\left(p_{j}^{\tau}-p_{j-1}^{\tau}\right)}{\Delta z}\right.\right. \\
- & \left.\frac{\left(h_{j}^{\tau}-h_{j-1}^{\tau}\right)}{\Delta z}+\left.\frac{1}{\rho_{j}^{\tau}} \frac{\partial p_{\tau}}{\partial z}\right|_{j} ^{\tau}\right)+\frac{4 \alpha_{j}^{\tau}\left(\theta_{j}^{\tau}-t_{j}^{\tau}\right)}{d_{i n} \rho_{j}^{\tau}}- \\
& \left.-\left.\frac{1}{A \rho_{j}^{\tau}} \frac{\partial p}{\partial \rho}\right|_{j} ^{\tau} \frac{\left(\dot{m}_{j}^{\tau}-\dot{m}_{j-1}^{\tau}\right)}{\Delta z}\right], \quad j=2, \ldots, M .
\end{aligned}
$$

The derivatives $\frac{\partial p}{\partial h}$ and $\frac{\partial p}{\partial \rho}$ in the Eq. (12) are calculated using the difference method. The fluid mass flow rate in the above formulae is referred to one waterwall pipe and it is calculated as:

$$
\dot{m}=\frac{\dot{m}_{t}}{n}
$$

where $\dot{m}_{t}$ is total fluid mass flow rate at the waterwalls inlet.

All the thermophysical properties of the fluid and waterwall pipe material are computed in the on-line mode with the use of appropriate functions and programs. To develop them, data published in $[2,12-16]$ were used.

In view of the iterative character of the suggested method, the computations should satisfy the following condition:

$$
\frac{\left|Y_{j,(k+1)}^{\tau+\Delta \tau}-Y_{j,(k)}^{\tau+\Delta \tau}\right|}{Y_{j,(k+1)}^{\tau+\Delta \tau}} \leq \vartheta
$$

where: $Y$ - currently evaluated temperature in node $j ; \vartheta$ - assumed tolerance of iteration; $k=1,2, \ldots$ - next iteration counter after a single time step.

The formulae allowing for in the on-line mode calculation of the heat transfer coefficient on the internal surface of pipes at overcritical pressures are presented in the literature [17-22]. By the review of these formulae and their analysis made in the paper [21], it can be seen that the most accurate results have been obtained with the use of the Bishop experimental formula [17]:

$$
\mathrm{Nu}=0.0069 \operatorname{Re}_{f}^{0.9} \overline{\operatorname{Pr}}_{f}^{0.66}\left(\frac{\rho_{w}}{\rho_{f}}\right)^{0.43}\left(1+2.4 \frac{d_{i n}}{z}\right),
$$

where Prandtl number $-\overline{\operatorname{Pr}_{f}}=\left(\frac{h_{w}-h_{f}}{\theta-t_{f}} \frac{\mu_{f}}{k_{f}}\right)$, and subscript $w$ refers to 
the wall. The quantities with the subscript $f$ should be calculated depending on the bulk temperature of the fluid for the turbulent flow:

$$
\bar{t}=\frac{\int \rho \cdot c_{f} \cdot w \cdot t \cdot d A}{\int \rho \cdot c_{f} \cdot w \cdot d A} .
$$

The formula (13) is valid for turbulent flow and for the following ranges:

- pressure: $p=22.8-27.6 \mathrm{MPa}$,

- fluid temperature: $t=282-527^{\circ} \mathrm{C}$,

- fluid mass flux: $G=651-3662 \mathrm{~kg} /\left(\mathrm{m}^{2} \mathrm{~s}\right)$,

- thermal load of waterwalls: $\dot{q}=0.31-3.46 \mathrm{MW} / \mathrm{m}^{2}$.

For the calculation of the heat transfer coefficient the Kitoh formula [19] is also often used:

$$
\mathrm{Nu}=0.015 \operatorname{Re}_{f}^{0.85} \operatorname{Pr}_{f}^{m},
$$

where

$$
m=0.69-\frac{81000}{q^{*}}+f_{c} \dot{q}, \quad q^{*}=200 G^{1.2}
$$

and

$$
f_{c}= \begin{cases}29 \times 10^{-8}+\frac{0.11}{q^{*}} & \text { for } \quad 0 \leq h_{f} \leq 1500 \mathrm{~kJ} / \mathrm{kg} \\ -8.7 \times 10^{-8}-\frac{0.65}{q^{*}} & \text { for } \quad 1500 \leq h_{f} \leq 3300 \mathrm{~kJ} / \mathrm{kg} \\ -9.7 \times 10^{-7}+\frac{1.3}{q^{*}} & \text { for } \quad 3300 \leq h_{f} \leq 4000 \mathrm{~kJ} / \mathrm{kg}\end{cases}
$$

Equation (15) was derived for the following ranges:

- fluid temperature: $t=20-550{ }^{\circ} \mathrm{C}$,

- fluid enthalpy: $h=100-3300 \mathrm{~kJ} / \mathrm{kg}$,

- fluid mass flux: $G=100-1750 \mathrm{~kg} /\left(\mathrm{m}^{2} \mathrm{~s}\right)$,

- thermal load of waterwalls: $\dot{q}=0.0-1.8 \mathrm{MW} / \mathrm{m}^{2}$.

The distribution of the heat load of waterwalls in the form of the heat flux $\dot{q}\left[\mathrm{~W} / \mathrm{m}^{2}\right]$ is known from the heat transfer calculations of the boiler combustion chamber. These calculations can be carried out with the use of the zone method [23]. 


\subsection{Method 2}

In this method another approach to solve the balance Eqs. (2), (4), and (8) is proposed. After some simplification and transformation of these equations, it is possible to bring them to the form, where on the left hand side spatial derivatives are obtained, whereas on the right side - time derivatives:

- mass balance:

$$
\frac{\partial \dot{m}}{\partial z}=-A \frac{\partial \rho}{\partial \tau}
$$

- momentum balance:

$$
\frac{\partial}{\partial z}\left(\frac{\dot{m}^{2}}{A^{2} \rho}+p\right)=-\frac{1}{A} \frac{\partial \dot{m}}{\partial \tau}-\frac{\partial p_{\tau}}{\partial z}-\rho g \sin \varphi,
$$

- energy balance:

$$
\frac{\partial h}{\partial z}=\frac{\rho A}{\dot{m}}\left(-\frac{\partial h}{\partial \tau}+\frac{4 \alpha(\theta-t)}{d_{i n} \rho}\right) .
$$

Time derivatives on the right hand side are replaced by a backward difference scheme. The obtained ordinary differential equation system is solved with the Runge-Kutta method.

After the modifications the energy balance equation is:

$$
\frac{d h_{j}^{\tau}}{d z}=\frac{\rho_{j}^{\tau-\Delta \tau} A}{\dot{m}_{j}^{\tau-\Delta \tau}}\left(-\frac{h_{j}^{\tau}-h_{j}^{\tau-\Delta \tau}}{\Delta \tau}+\frac{4 \alpha_{j}^{\tau-\Delta \tau}\left(\theta_{j}^{\tau-\Delta \tau}-t_{j}^{\tau-\Delta \tau}\right)}{d_{i n} \rho_{j}^{\tau-\Delta \tau}}\right) .
$$

After solving the mass and momentum conservation equations, the following formulae were obtained, respectively:

$$
\begin{gathered}
\frac{d \dot{m}_{j}^{\tau}}{d z}=-A \frac{\rho_{j}^{\tau}-\rho_{j}^{\tau-\Delta \tau}}{\Delta \tau} \\
\frac{d}{d z}\left(\frac{\left(\dot{m}^{2}\right)_{j}^{\tau}}{A^{2} \rho_{j}^{\tau}}+p_{j}^{\tau}\right)=-\frac{1}{A} \frac{\left(\dot{m}^{2}\right)_{j}^{\tau}-\left(\dot{m}^{2}\right)_{j}^{\tau-\Delta \tau}}{\Delta \tau}-\frac{\xi\left(\dot{m}^{2}\right)_{j}^{\tau}}{2 d_{i n} A^{2} \rho_{j}^{\tau}}-\rho_{j}^{\tau} g \sin \varphi
\end{gathered}
$$

In Eqs. (21)-(23) the subscript $j$ denotes the number of analysed cross sections, and it varies from 2 up to $M(j=2, \ldots, M)$.

As in method 1, all the thermophysical properties of the fluid and waterwall pipe, as well as the heat transfer coefficient, are calculated in real-time. 
In both presented methods, the following condition should be obeyed (the Courant-Friedrichs-Lewy stability condition over the time step) [24]:

$$
\Delta \tau \leq \frac{\Delta z}{w} .
$$

When satisfying this condition, the numerical solution is reached with a speed $\Delta z / \Delta \tau$, greater than the physical speed $w$.

In the proposed methods the computations are carried out in the direction of the fluid flow in one tube. The tube is equal in size to those existing in the real object. The mass flow rate of fluid is also related to a single tube. The proposed methods are based on the assumption that the operating fluid flows uniformly through all tubes of the combustion chamber waterwalls. The thermal load distribution along the combustion chamber height is known from the calculations of the heat exchange in the chamber. These calculations are carried out with the use of the zone method.

\section{Computational verification}

In order to verify the methods proposed in Section 3, test calculations are presented. The calculations refer to a lignite coal-fired boiler. For the numerical analyses, the data refer to a boiler operating in one of the European power plants were assumed (nominal boiler power: $N=670 \mathrm{MW}$; live steam pressure: $p=28.8 \mathrm{MPa}$; live steam temperature: $t=600{ }^{\circ} \mathrm{C}$ ). The computations were carried out with the use of the Fortran code [25].

The verification consists of the comparison of the results obtained using the methods described in Section 3. Additionally, in order to determine the actual distributions of both the fluid and waterwall pipe temperature, a computations by means of the CFD were carried out. To compare CFD results with the results obtained by methods 1 and 2, the bulk temperature of fluid in selected cross-sections was also computed. Figure 2 shows the scheme of the analysed combustion chamber and its division into zones.

For the calculation, the following data were assumed:

- mass flow rate: $\dot{m}_{t}=2000 \mathrm{t} / \mathrm{h}$,

- water pressure at the waterwall inlet: $p=30 \mathrm{MPa}$,

- thermal load of the combustion chamber: $\dot{q}=150000 \mathrm{~W} / \mathrm{m}^{2}$,

- inclination angle of waterwall pipes: $\varphi=22^{\circ}$, 


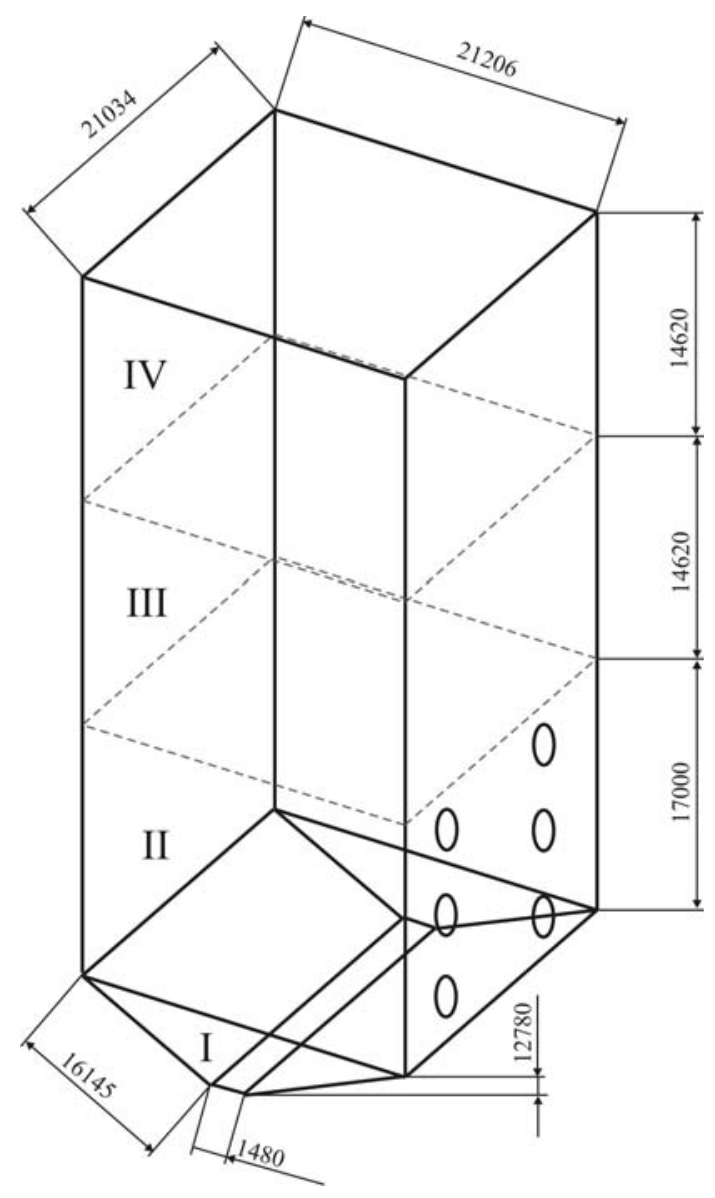

Figure 2. Division of the boiler combustion chamber into zones.

- number of waterwall pipes: $n=735$,

- pipes length: $l=123 \mathrm{~m}$,

- pipe outer diameter $\times$ pipe wall thickness: $d_{o} \times g_{w}=38 \times 7.1 \mathrm{~mm}$,

- pipe layout pitch: pitch $=61 \mathrm{~mm}$,

- waterwall pipe material: 13CrMo4-5 and 7CrMoVTiB10-10,

- supplied water temperature at the inlet to the combustion chamber waterwalls: $t=290^{\circ} \mathrm{C}$. 
It was assumed that water, at the initial temperature $t=290^{\circ} \mathrm{C}$, flows through a $\phi 38 \times 7.1 \mathrm{~mm}$ pipe made of $13 \mathrm{CrMo} 4-5$ steel and $7 \mathrm{CrMoVTiB} 10-10$ steel respectively for lower and upper sections of combustion chamber waterwalls. The initial temperature of the pipe wall of $l=123 \mathrm{~m}$ in length at the time $\tau=0$ was the same. Beginning from the next time step, the heat step function $(\Delta q=\dot{q} \cdot p i t c h)$ at the outer surface of the pipe was assumed. The constant thermal load along the combustion chamber height was taken into consideration.

In order to achieve a stable solution of the difference equations, optimal space and time steps were determined. These steps were evaluated based on a comparison of the results obtained using the proposed method 1 with the results of analytical solutions for transient states. A step function of the heating on the external surface of the tube was analysed [26]. As the results, $\Delta \tau=0.04 \mathrm{~s}$ and $\Delta z=0.5 \mathrm{~m}$ were obtained. These steps allow dissipation and dispersion at the grid to be avoided and at the same time allow the Courant condition (21) to be satisfied. The computations were carried out with an assumed iteration tolerance $\vartheta=10^{-6}$.

The calculations and comparisons results for the selected cross-sections are shown in Figs. 3-10.

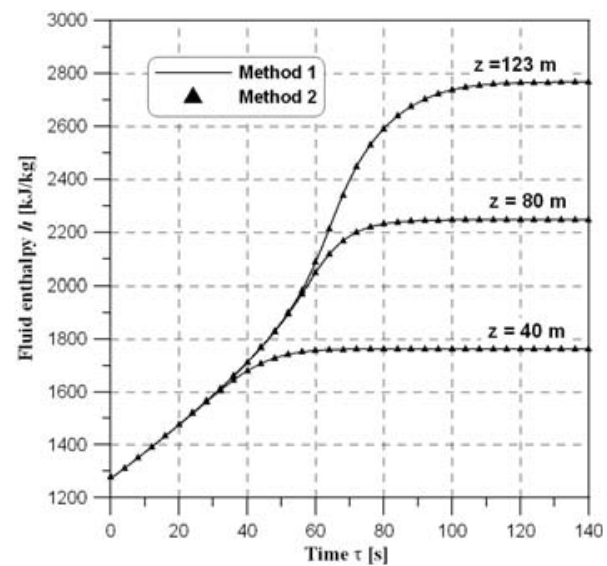

Figure 3. Comparison of the fluid enthalpy histories.

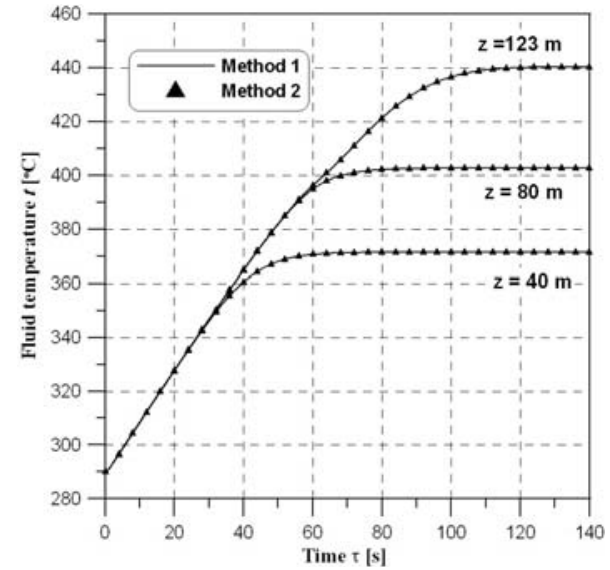

Figure 4. Comparison of the fluid temperature histories.

Figures $3-5$ indicate completely satisfactory convergence of the histories obtained by both numerical methods described in Section 3. The histories shown in other figures are obtained with the use of the method 1. An anal- 


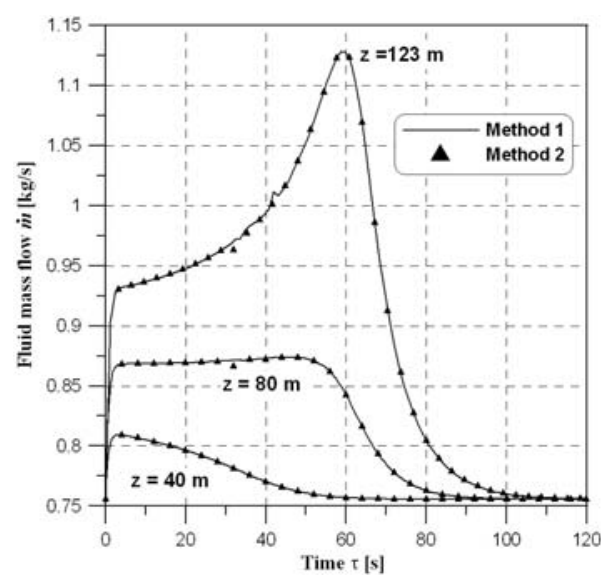

Figure 5. Comparison of the fluid mass flow rate histories.

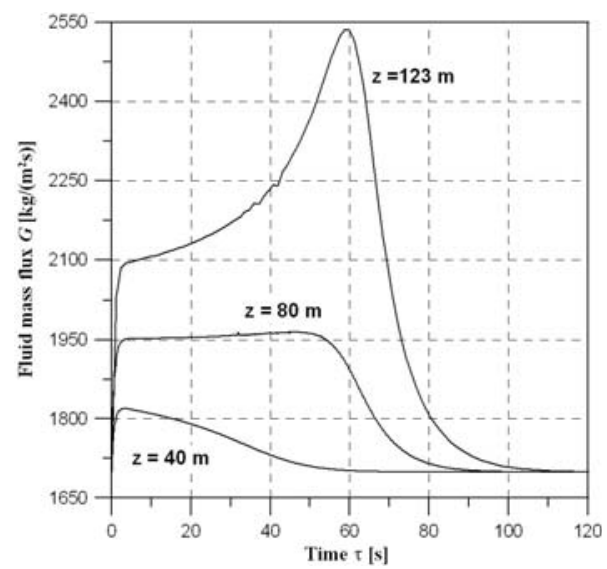

Figure 7. Histories of the fluid mass flux in selected cross-sections.

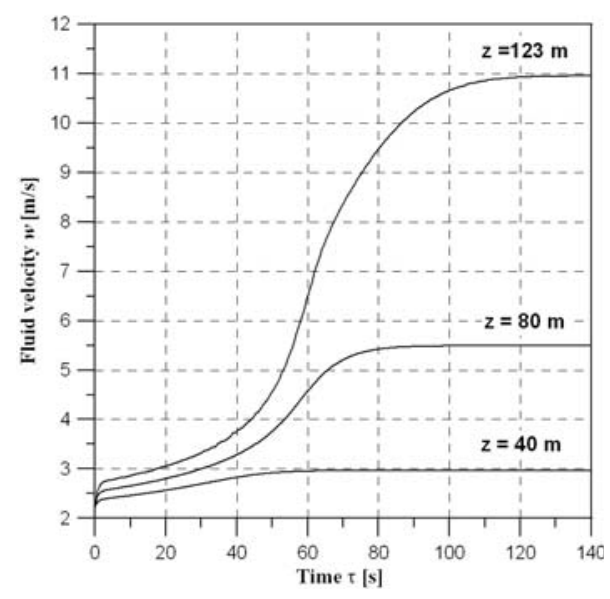

Figure 6. Histories of the fluid velocity in selected cross-sections.

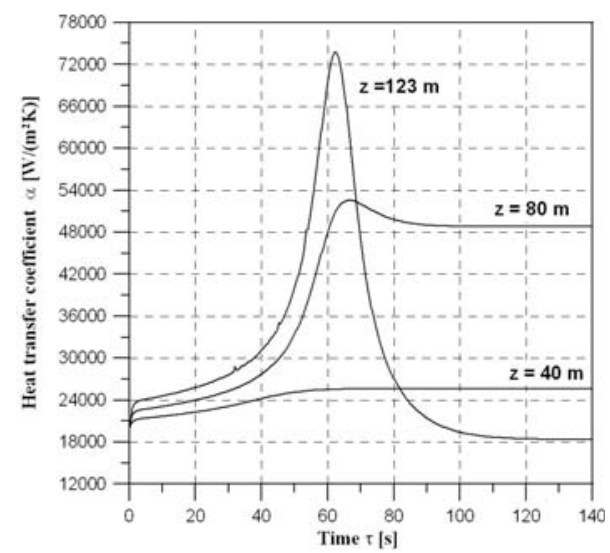

Figure 8. Histories of the heat transfer coefficient in selected crosssections.

ysis of the Fig. 7 shows, that fluid mass flux reaches values recommended for once-through boiler with screw arrangement of pipes. For lignite these values should be at the level of $2000 \mathrm{~kg} /\left(\mathrm{m}^{2} \mathrm{~s}\right)$ [27]. As an example of the thermophysical properties calculated in the on-line mode, the obtained histories of fluid density and specific heat are shown in Figs. 9 and 10. 


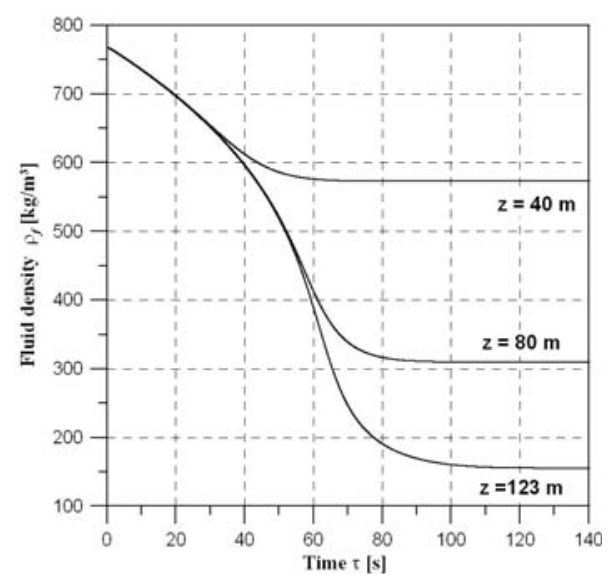

Figure 9. Histories of the fluid density in selected cross-sections.

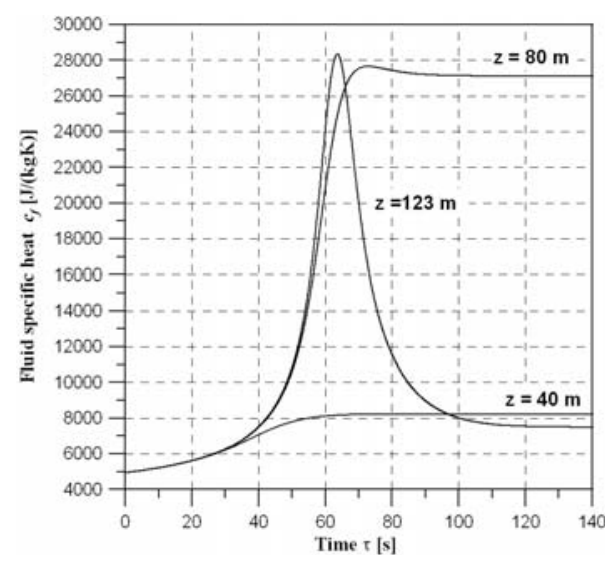

Figure 10. Histories of the fluid specific heat in selected cross-sections.

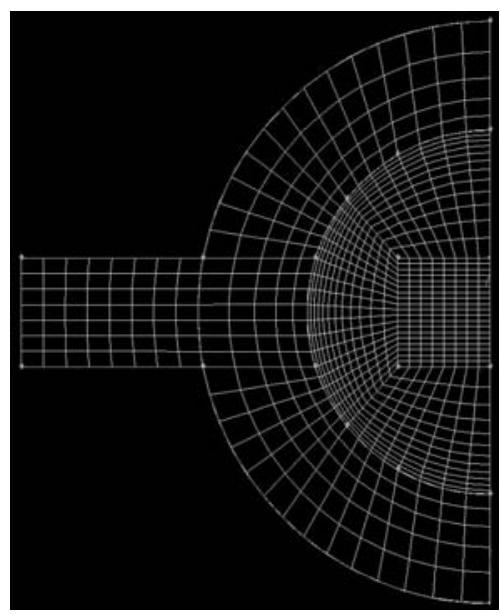

Figure 11. Developed mesh for the needs of CFD analysis.

In order to obtain the real temperature distributions of the fluid and waterwall pipe with the fin, in the analysed cross-sections, CFD computations were carried out. These computations were performed with the same initial and boundary conditions as for the case described above. The variability of the thermophysical properties was taken into consideration. The assumed mesh is shown in Fig. 11 and selected calculation results are shown in Fig. 12. 


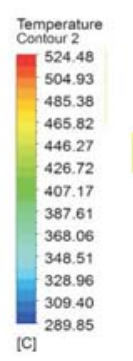

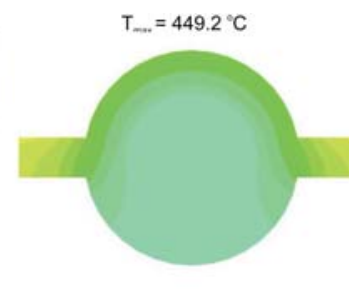

$40 \mathrm{~m}$
$T_{\max }=479.2^{\circ} \mathrm{C}$

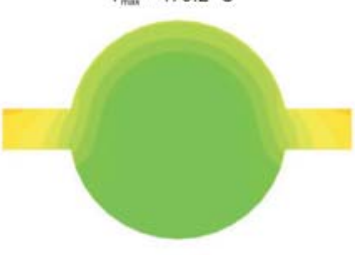

$80 \mathrm{~m}$

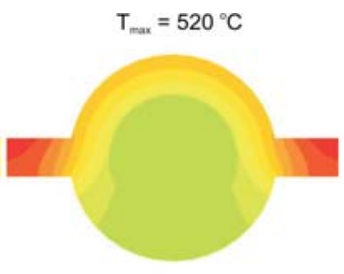

$123 \mathrm{~m}$

Figure 12. Temperature distribution for selected cross-sections obtained from the CFD (steady state conditions).

Table 1 presents a comparison of the fluid bulk temperature obtained from CFD and with the use of the method 1. These results refer to the steady state conditions, which occurred after $\sim 120 \mathrm{~s}$.

Table 1. Comparison of the fluid bulk temperature in selected cross-sections (for the steady state).

\begin{tabular}{|c|c|c|}
\hline \multirow{2}{*}{$l[\mathrm{~m}]$} & \multicolumn{2}{|c|}{$t\left[{ }^{\circ} \mathrm{C}\right]$} \\
\cline { 2 - 3 } & CFD & Method 1 \\
\hline \hline 5 & 301.8 & 301.9 \\
\hline 10 & 311.7 & 312.4 \\
\hline 20 & 331.9 & 333.3 \\
\hline 30 & 354.7 & 354.0 \\
\hline 40 & 372.9 & 370.6 \\
\hline 50 & 384.4 & 383.5 \\
\hline 55 & 388.7 & 388.4 \\
\hline 60 & 392.4 & 392.4 \\
\hline 65 & 395.6 & 395.5 \\
\hline 70 & 398.5 & 398.1 \\
\hline 75 & 401.1 & 400.3 \\
\hline 80 & 403.5 & 402.7 \\
\hline 85 & 406.2 & 404.9 \\
\hline 90 & 409.2 & 407.5 \\
\hline 95 & 412.5 & 410.4 \\
\hline 100 & 415.3 & 413.9 \\
\hline 105 & 419.7 & 418.1 \\
\hline 110 & 424.9 & 423.1 \\
\hline 115 & 432.3 & 430.3 \\
\hline 123 & 440.2 & 440.3 \\
\hline
\end{tabular}

When analysing the results shown in the Tab. 1, it can be seen that a fully satisfactory agreement of the calculation results obtained with the use 
of the method 1 and obtained by CFD was achieved. From Fig. 12 follows, that the maximum recommended design metal temperature is not exceeded. This temperature is $560{ }^{\circ} \mathrm{C}$ and $580{ }^{\circ} \mathrm{C}$ respectively for $13 \mathrm{CrMo} 4-5$ and 7CrMoVTiB10-10 steel.

\section{Conclusions}

In this paper, a mathematical model for the simulation of heat-flow phenomena occurring on the side of the fluid in the waterwalls of boiler combustion chamber for supercritical parameters is proposed. It is a one-dimensional model with distributed parameters, based on the solution of equations describing the laws of mass, momentum, and energy conservation. The fluid density and temperature are determined in the on-line mode as the function of its enthalpy and pressure. All thermophysical properties of the fluid and waterwall pipe material are calculated in the real-time. The correctness of the determination of these properties in the on-line mode, especially for the fluid in the critical point area, is of great importance for the accuracy of the obtained temperature distributions. The distribution of the heat transfer coefficient is determined in the on-line mode, also.

Two methods of solution of the derived equations are proposed. In the first one the implicit finite difference scheme is used. The second proposed approach of the derived equations solution is the Runge-Kutta method.

The computational verification of the developed methods was carried out. Having the basic data, with regard to supercritical parameters for the combustion chamber of lignite coal-fired boiler, the calculations were carried out using both developed methods. The results were compared to each other and completely satisfactory convergence was achieved. The distribution of waterwall pipe temperature was determined by means of the CFD. Calculations were carried out for the coiled pipes. Comparing the fluid temperature in selected cross-sections, obtained by means of the CFD, with the results obtained using method 1 a satisfactory convergence was found. This shows that the proposed numerical methods (1 and 2) are indeed effective. In these methods it is also possible to take into consideration the thermal load varying along the height of boiler combustion chamber. 


\section{References}

[1] TAler J.: Dynamics of Steam Generators with Natural Circulation Accounting Thermal Stresses. Cracow University of Technology, Cracow 1987 (in Polish).

[2] Taler J., Duda P.: Solving Direct and Inverse Heat Conduction Problems, Springer, Berlin 2006.

[3] Zima W.: Mathematical model of transient processes in steam superheaters. Forschung im Ingenieurwesen 68, Springer, Berlin Heidelberg 2003, 51-59.

[4] Zima W.: Simulation of dynamics of a boiler steam superheater with an attemperator. Proceedings of the Institution of Mechanical Engineers, Part A: Journal of Power and Energy 220(2006), 793-801.

[5] Zima W.: Mathematical modelling of transient processes in convective heated surfaces of boilers. Forschung im Ingenieurwesen 71, Springer, Berlin Heidelberg 2007, $113-123$.

[6] Grądziel S., Zima W., Cebula A.: Modelling of the transient heat transfer occurring on heated surfaces of boilers. Archives of Energetics 1-2(2007), 53-60 (in Polish).

[7] Profos P.: Dynamics of Superheater Control. Combustion 31(1959).

[8] Profos P.: Die Regelung von Dampfanlagen. Springer Verlag, Berlin 1962 (in Ger$\operatorname{man})$.

[9] Krzyżanowski J.A., G七uch J.: Heat-flow diagnostics of energy objects. IMP PAN, Gdansk 2004 (in Polish).

[10] Fluent 6.0. Computational fluid dynamics software. Fluent Inc., Lebanon, NH, USA 2006.

[11] Bertin J. J.: Aerodynamics for Engineers, 4th edn. Prentice Hall, New Jersey 2002.

[12] Thermodynamic and Transport Properties of Steam. ASME Steam Tables, 6th edn., USA 1993.

[13] Properties of water and steam using the $196^{\text {r }}$ IFC formulation for industrial use. American Society of Mechanical Engineers, 1992.

[14] VDI-Warmeatlas. Springer-Verlag Berlin and Heidelberg GmbH \& Co. K., 2006.

[15] Water \& Steam. IAPWS-IF97, Springer-Verlag, 1999.

[16] Wegst C.W.: Stahlschlüssel. Verlag Stahlschlüssel Wegst GMBH, 2001 (in German).

[17] Bishop A.A., Sandberg R.O., Tong L.S.: Forced convection heat transfer to water at near-critical temperature and supercritical pressures. Joint Meeting of the American Institute of Chemical Engineers and the British Institution of Chemical Engineers, London 1964.

[18] Griem H.: A new procedure for the prediction of forced convection heat transfer at near- and supercritical pressure. Heat and Mass Transfer 31(1996), 301-305.

[19] Кiтон K., Koshizuka S., Oka Yo.: Refinement of transient criteria and safety analysis for a high temperature reactor cooled by supercritical water. In: Proceedings of the Seventh International Conference on Nuclear Engineering (ICONE-7), Tokyo, April 19-23, 1999, Paper No. 7234. 
[20] Loewenberg M.F., Laurien E., Class A., Schulenberg T.: Supercritical water heat transfer in vertical tubes. Progress in Nuclear Energy 50(2008), 532-538.

[21] YU J., JiA B., WU D., WANG D.: Optimization of heat transfer coefficient correlation at supercritical pressure using genetic algorithms, Heat Mass Transfer 45(2009), $757-766$.

[22] Yu J., LiU H., JiA B.: Sub-channel analysis of CANDU-SCWR and review of heat transfer correlations. Progress in Nuclear Energy 51(2009), 246-252.

[23] Kuznetsov N.W., Nitor W.W., Dubovski I.E. and Karasina E.S.: Thermal Calculations of Steam Boilers. Standard Method, Energy, Moscow 1973 (in Russian).

[24] Gerald C.F., Wheatley P.O.: Applied Numerical Analysis. Addison-Wesley Publishing Company, New York 1994.

[25] Fortran PowerStation 4.0. Microsoft Developer Studio, Microsoft Corporation 1994-95.

[26] Serov E.P., Korolkov B.P.: Dynamics of steam generators. Energia, Moscow 1981 (in Russian).

[27] Ortowski P., Dobrzański W., Szwarc E.: Steam boilers - structure and calculations. WNT, Warsaw 1979 (in Polish). 